\title{
Octogenarians may benefit from stage-specific small cell lung cancer treatment
}

\author{
Bin Zhou ${ }^{1 \#}$, Qiuyuan $\mathrm{Li}^{1 \#}$, Linlin Qin ${ }^{1 \#}$, Zhao $\mathrm{Li}^{1 \#}$, Kaiqi Jin ${ }^{1}$, Jie Dai ${ }^{1}$, Yuming Zhu ${ }^{1}$, Yang Yang ${ }^{1}$, \\ Salma K. Jabbour ${ }^{2}$, Alfredo Tartarone ${ }^{3}$, Calvin S. H. Ng ${ }^{4}$, Alfons Navarro ${ }^{5}$, Cecilia Pompili ${ }^{6}$, Gening Jiang ${ }^{1}$ \\ ${ }^{1}$ Department of Thoracic Surgery, Shanghai Pulmonary Hospital, Shanghai Tongji University School of Medicine, Shanghai, China; ${ }^{2}$ Department \\ of Radiation Oncology, Rutgers Cancer Institute of New Jersey, New Brunswick, NJ, USA; ${ }^{3}$ Division of Medical Oncology, Department of Onco- \\ Hematology, IRCCS-CROB, Referral Cancer Center of Basilicata, Rionero in Vulture (PZ), Italy; ${ }^{4}$ Division of Cardiothoracic Surgery, Department \\ of Surgery, Prince of Wales Hospital, the Chinese University of Hong Kong, Hong Kong, China; ${ }^{5}$ Molecular Oncology and Embryology Laboratory, \\ Human Anatomy Unit, Faculty of Medicine and Health Sciences, University of Barcelona, Barcelona, Spain; ${ }^{6}$ Department of Thoracic Surgery, \\ University of Leeds, Section of Patient Centred Outcomes Research, Leeds Institute for Medical Research at St. James's, St. James' Institute of \\ Oncology, Leeds, UK \\ Contributions: (I) Conception and design: B Zhou, Q Li, L Qin, Z Li; (II) Administrative support: Y Yang, G Jiang; (III) Provision of study materials \\ or patients: B Zhou; (IV) Collection and assembly of data: B Zhou, Q Li; (V) Data analysis and interpretation: B Zhou, Q Li; (VI) Manuscript \\ writing: All authors; (VII) Final approval of manuscript: All authors. \\ \#These authors contributed equally to this work. \\ Correspondence to: Gening Jiang, MD. Department of Thoracic Surgery, Shanghai Pulmonary Hospital, Shanghai Tongji University School of \\ Medicine, 507 Zhengmin, Shanghai 200082, China. Email: geningjiang@tongji.edu.cn.
}

Background: Our study investigates treatment profiles in octogenarian patients with small cell lung cancer (SCLC) and assesses each treatment's role in a stage-specific manner.

Methods: Patient data from individuals with SCLC aged 80 years and older between 1988 and 2015 in the Surveillance, Epidemiology, and End Results Program (SEER) database were extracted. Cancer-specific survival (CSS) between patients with no treatment and different treatment groups were compared by the Kaplan-Meier method, with stratifications by stage. Cox Proportional Hazard model further identified independent prognostic factors.

Results: A total of 7,290 patients were included in this study. Notably, 3,358 (46.1\%) patients did not receive active treatment. Compared with the no active treatment group, the CSS of patients who received treatment was significantly improved (median 6 vs. 0 months, $\mathrm{P}<0.001$ ) and further validated in stage subgroups. Chemotherapy combined with local therapy was associated with the best CSS in regional and distant disease stages, with the hazard ratios (HR) and 95\% confidence intervals (CI) being $0.30(0.26-0.34)$ and $0.27(0.25-0.30)$, respectively. Local therapy only appeared to confer better oncological outcomes $(\mathrm{HR}=0.33$; 95\% CI: $0.25-0.42)$ than chemotherapy only (HR $=0.37$; 95\% CI: $0.29-0.47)$ in the localized disease stage.

Conclusions: Although nearly half of octogenarians with SCLC did not receive active treatment in the real clinical setting, these patients may benefit from treatment. Chemotherapy combined with local therapy may provide the best treatment choice in octogenarians with advanced SCLC, while local therapy appears to play a more critical role in treating those with early-stage disease.

Keywords: Small cell lung cancer (SCLC); octogenarians; stage; local therapy; chemotherapy

Submitted Jul 20, 2021. Accepted for publication Oct 22, 2021.

doi: $10.21037 /$ tlcr-21-839

View this article at: https://dx.doi.org/10.21037/tlcr-21-839 


\section{Introduction}

Lung cancer is one of the most common malignancies and the leading cause of cancer-related death (1). Small-cell lung cancer (SCLC) accounts for approximately $15 \%$ of primary lung cancer globally with an estimated annual incidence of 22,000 to 34,000 cases per year, and it is strongly associated with tobacco abuse (2). Early metastasis characterizes SCLC with a median survival time of $15-20$ months in limitedstage SCLC (LS-SCLC) and 8-13 months in extensivestage SCLC (ES-SCLC) (3).

However, at the time of diagnosis of lung cancer, patients are not often young, with an average age of about 70 years old (4). As the population ages, there are likely to be an increasing number of diagnosed patients at an older age. Therefore, clinicians must carefully balance the benefits and risks of therapeutic interventions when designing a treatment plan in elderly patients, who may have multiple comorbidities and potentially limited life expectancies. Previous studies show that elderly patients with non-small cell lung cancer (NSCLC) have distinctive disease characteristics and treatment profiles compared to younger patients (5-9). A Surveillance, Epidemiology, and End Results Program (SEER) database analysis found that radiotherapy has replaced surgery as the most common used modality in early-stage NSCLC in patients $\geq 80$ years in the United States and an improvement was observed in cancer-specific survival (CSS) and overall survival (OS) for patients treated with definitive RT and surgery (10). Although evidence is more limited for SCLC compared with NSCLC, the same rules may likely apply. However, the survival of SCLC has shown only modest improvements despite overall improvements in outcomes for other cancer types. Since, the proportion of elderly patients continues to expand (11); a detailed analysis of this specific cohort with octogenarians regarding their treatment options and the respective outcome is of paramount importance. Several retrospective studies demonstrated the benefit of treatment in a select subset of octogenarians with SCLC in forms of uni- as well as multi-modality therapy $(12,13)$. Chemotherapy or multimodality therapy is underused for elderly patients compared to younger patients with SCLC, but these could significantly improve the survival $(12,13)$. In this study, we aimed to augment the available literature by describing the treatment profile and analyzing the treatment outcomes in a stage-specific pattern for octogenarians with SCLC from the SEER dataset to identify the role of each treatment and optimal management strategy in this specific cohort.

We present the following article in accordance with the STROBE reporting checklist (available at https://dx.doi. org/10.21037/tlcr-21-839).

\section{Methods}

\section{Study population}

The SEER dataset was queried from 1988 to 2015 for patients with SCLC using the software SEER*Stat (version 8.3.6). The study was conducted in accordance with the Declaration of Helsinki (as revised in 2013). The Institution Review Board of Shanghai Pulmonary Hospital approved this study. Cases were limited to those with no history of other primary tumors and ages $\geq 80$ years. Histologic type ICD-O-3 of SCLC was identified as 8002 and 8041-8045. Any patients with unknown gender, race, stage, treatment methods, or follow-up information were excluded. SEER historical stage (see https://seer.cancer.gov/tools/ssm/) was adopted, which combined the clinical and pathological documentation of the extent of disease.

We stratified the study population based on whether they received active treatment or not, which was defined as a cancer-directed therapy, including surgery, radiotherapy, or chemotherapy. Among these, surgery and radiotherapy were further classified as local therapies.

\section{Statistical analysis}

Patient characteristics are summarized in Table 1. Continuous variables were examined using an independent sample Student's $t$-test. Categorical variables were compared by Pearson Chi-square test.

Survival curves were plotted by the Kaplan-Meier method and compared by the Log-rank test. The "BH" method was used to control the false discovery rate for pairwise comparison of survival differences between multiple groups (14). The independent prognostic factors were determined by the multivariate (MV) Cox Proportional Hazard model, controlling for age, sex, race, stage, and treatment methods.

All the analyses were done in $\mathrm{R}$ software (version 4.0.2, Institute for Statistics and Mathematics, Vienna, Austria; www.r-project.org) with necessary packages including "survival" and "survminer." Two-tailed $\mathrm{P}$ value $<0.05$ was 
Table 1 Patient characteristics

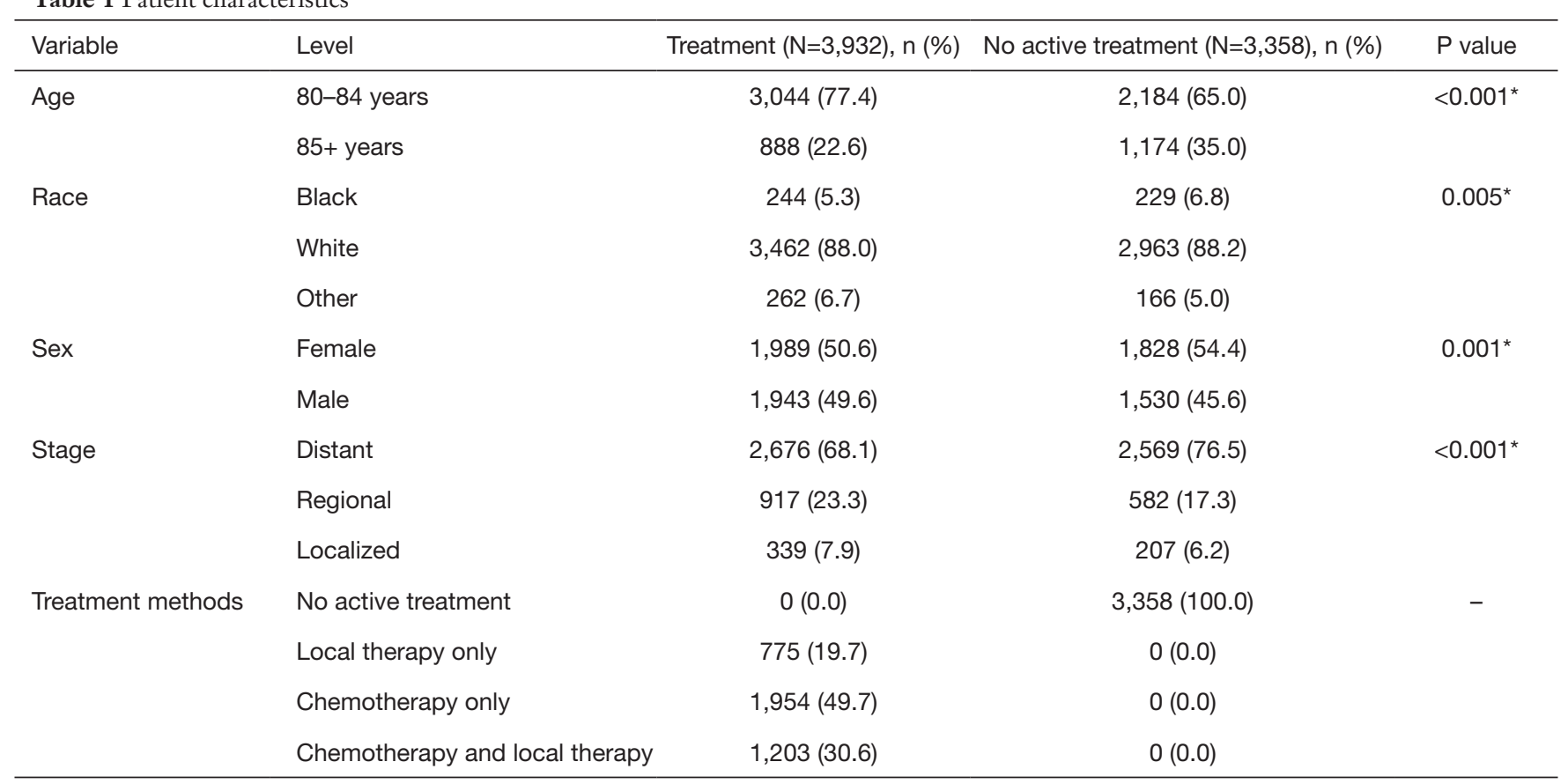

*, significant $P$ values.

considered statistically significant.

\section{Results}

\section{Patient characteristics}

There were 7,290 patients included in this study. Patient characteristics are summarized in Table 1. Notably, 3,358 (46.1\%) octogenarians with SCLC did not receive active treatment. Compared with the treatment group, octogenarians with SCLC who received no active treatment had an older age and more advanced stage (both $\mathrm{P}<0.001$ ). The proportion of patients with no active treatment remained static from 1988 to 2015 (Figure 1). The mean percentage of non-treated patients in the analyzed period was $46.1 \%$ (range, 34.2-50.2\%). Collectively, the percentages of patients who received no active treatment remained high across all stages, albeit smaller in the locoregional stages (38.0\% for localized, $38.9 \%$ for regional, and $49.0 \%$ for distant stage) (Figure 2).

\section{Survival analysis by stage and treatment methods}

CSS is shown in Figures 3,4. Patients with active treatment had a significantly better CSS than those who did not
(Figure $3 A, \mathrm{P}<0.0001$, median survival time 6 vs. 0 months). This was also the case for patients with different stages, with the median CSS being 13 vs. 4 months (Figure 3B), 9 vs. 1 month (Figure 3C), 4 vs. 0 months (Figure 3D) in localized, regional, and distant stages, respectively (all $\mathrm{P}<0.001)$. After we further broke down active treatment, it was observed that octogenarians benefited most from chemotherapy combined with local therapy in the regional and distant stages (Figure $4 C, 4 D$, all $\mathrm{P}<0.001$ ). However, in the localized stage, local therapy alone appeared to confer the best CSS, although a significant difference was only achieved for the comparison with chemotherapy $(\mathrm{P}<0.001)$ but not with the combination of chemotherapy and local therapy (Figure $4 B, \mathrm{P}=0.28$ ).

\section{Independent prognostic factors for CSS}

Univariate (UV) and multivariate (MV) analyses for independent prognostic factors for CSS are shown in Table 2. In UV analysis, age over 85 years old (HR $=1.23,95 \% \mathrm{CI}$ : $1.17-1.29, \mathrm{P}<0.001)$ and male sex $(\mathrm{HR}=1.06,95 \% \mathrm{CI}$ : $1.00-1.10, \mathrm{P}=0.02)$ were statistically significant risk factors, while regional $(\mathrm{HR}=0.57,95 \% \mathrm{CI}: 0.54-0.61, \mathrm{P}<0.001)$ or localized disease $(\mathrm{HR}=0.39,95 \% \mathrm{CI}: 0.35-0.43, \mathrm{P}<0.001)$ and active treatment $(\mathrm{HR}=0.36,95 \% \mathrm{CI}: 0.34-0.38$, 


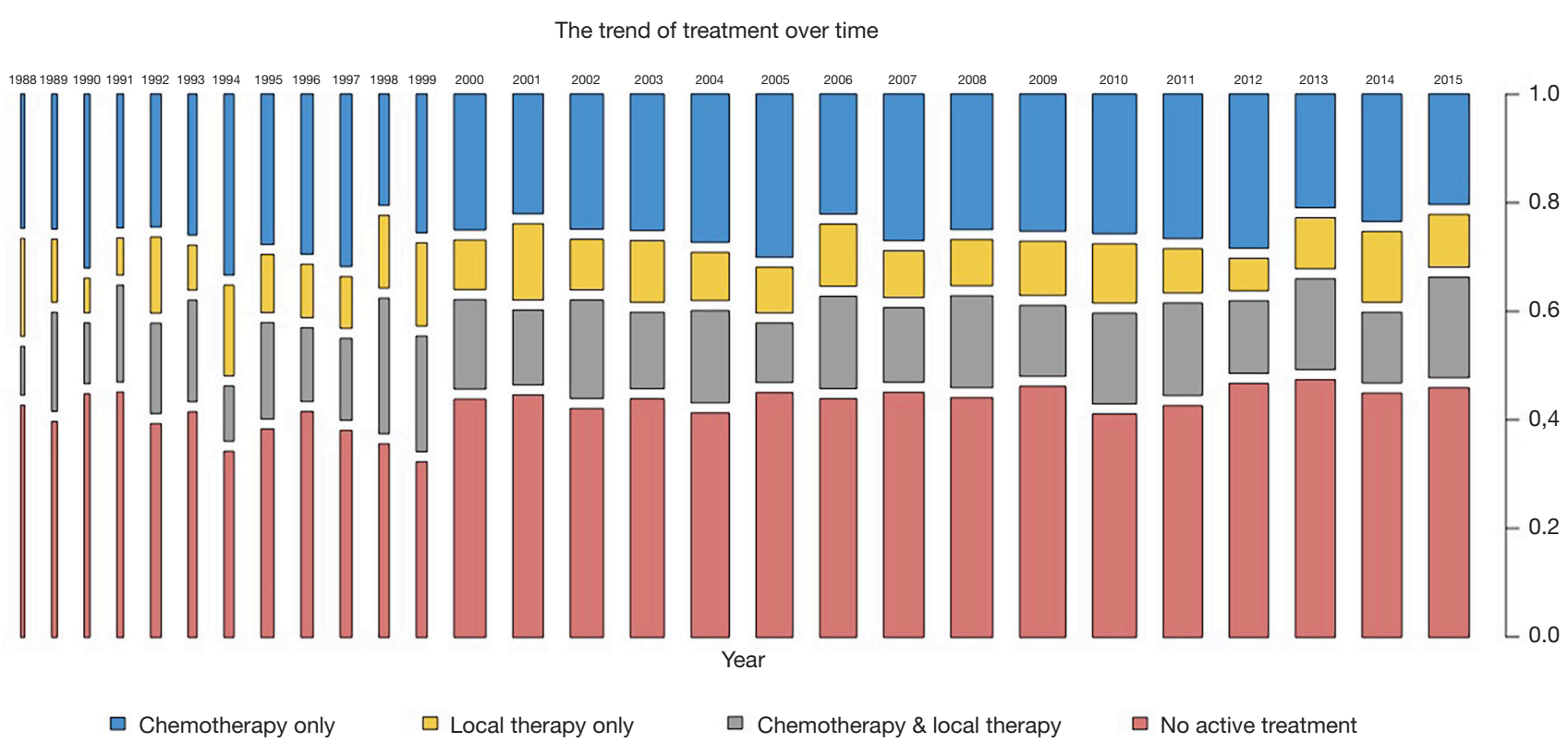

Figure 1 The trend of treatment over time. Colors correspond to different treatment methods. The length of the bar corresponds to the proportion of each treatment method in each year, and the width corresponds to the relative number of patients.

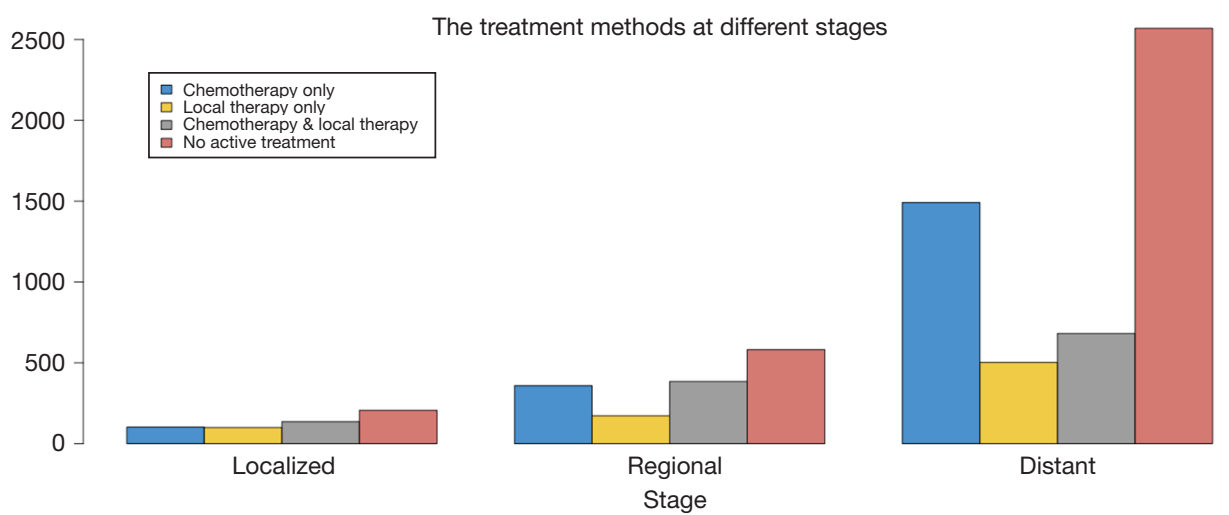

Figure 2 Treatment methods in different stages. Patients with no treatment had the biggest number in all stages.

$\mathrm{P}<0.001)$ were statistically significant prognostic factors. However, the $M V$ analysis only identified males $(\mathrm{HR}=1.14$, 95\% CI: $1.09-1.19, \mathrm{P}<0.001$ ), regional (HR $=0.58,95 \%$ CI: $0.55-0.62, \mathrm{P}<0.001)$ or localized disease $(\mathrm{HR}=0.35$, 95\% CI: $0.32-0.39, \mathrm{P}<0.001)$ as independent predictors for CSS. Compared to no treatment, each treatment method contributed significantly to survival with a significantly lower HR (all $\mathrm{P}<0.001)$ in both $\mathrm{UV}$ and MV analysis.

\section{Stage-specific subgroup analysis}

In subgroup MV analysis, all types of treatment showed consistent benefits to patient survival (all $\mathrm{P}<0.001$ ) in different stage groups (Table 3). Of note, for chemotherapy or chemotherapy and local therapy, there appeared to be a trend of increasing effects as the stage progressed. The survival advantage was most pronounced in the distant stage $(\mathrm{HR}=0.33,95 \% \mathrm{CI}: 0.30-0.35$ for chemo only; HR $=0.27,95 \%$ CI: $0.25-0.30$ for combined chemo) and least pronounced in the localized stage ( $\mathrm{HR}=0.58,95 \% \mathrm{CI}$ : 0.46-0.74 for chemo only; HR $=0.37,95 \%$ CI: $0.29-0.47$ for combined chemo). Noteworthy, the HR of local therapy showed an almost inverse trend, with the best treatment effect shown in the localized disease (HR $=0.33,95 \%$ CI: 

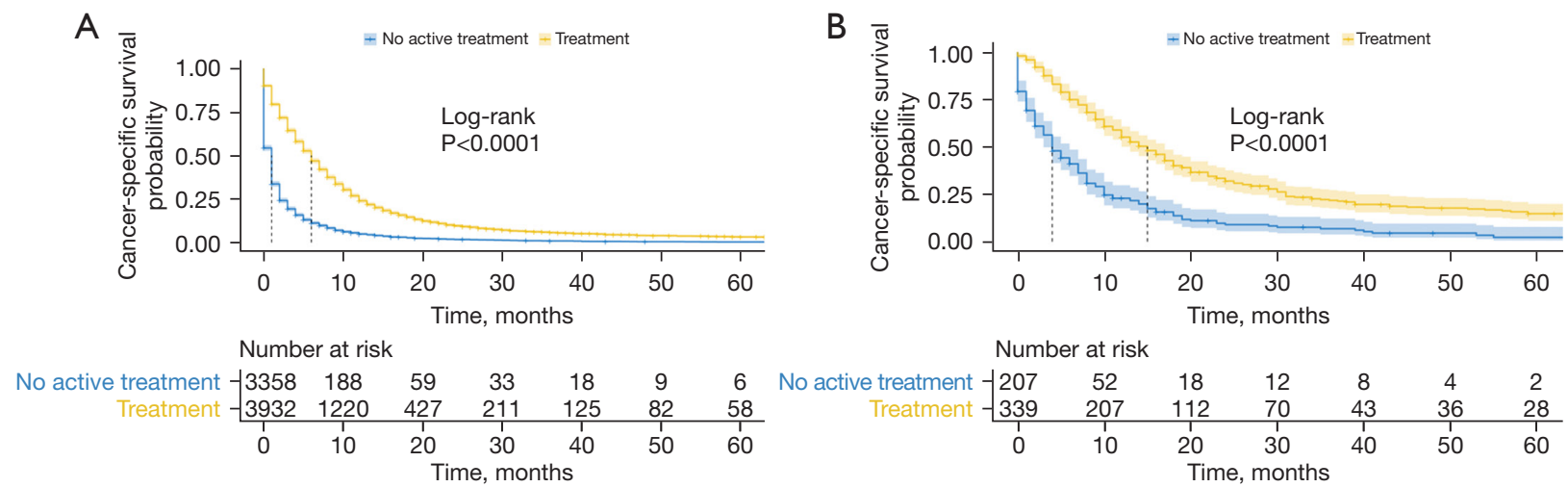

Number at risk
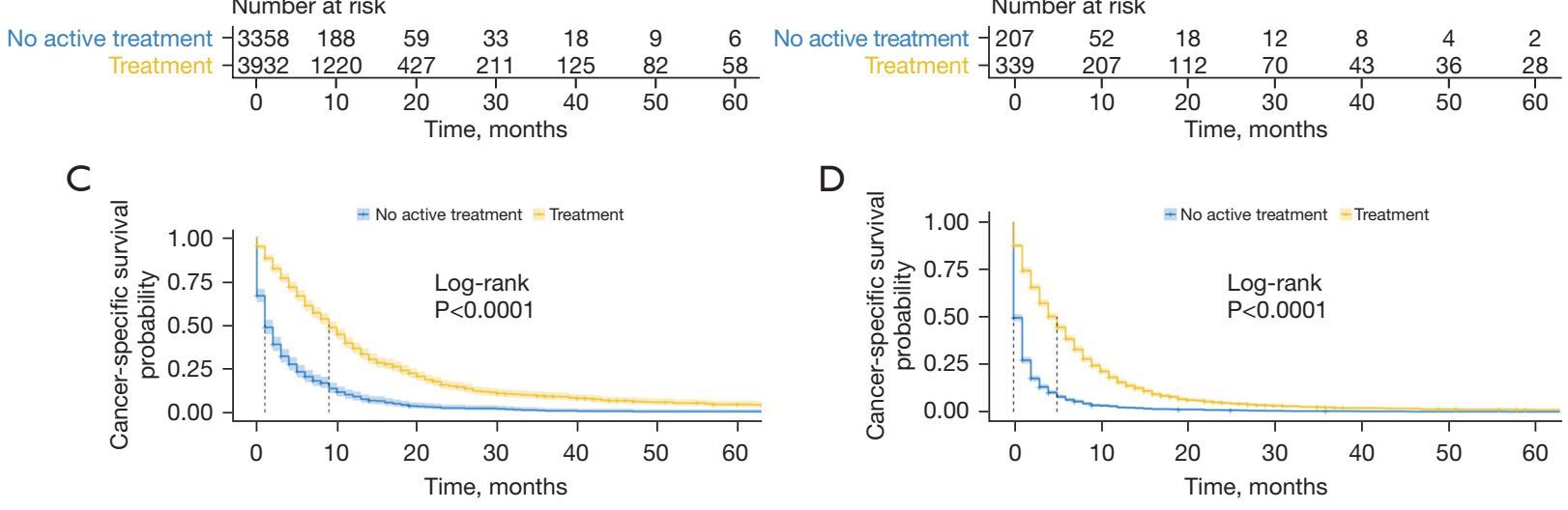

$\mathrm{D}$

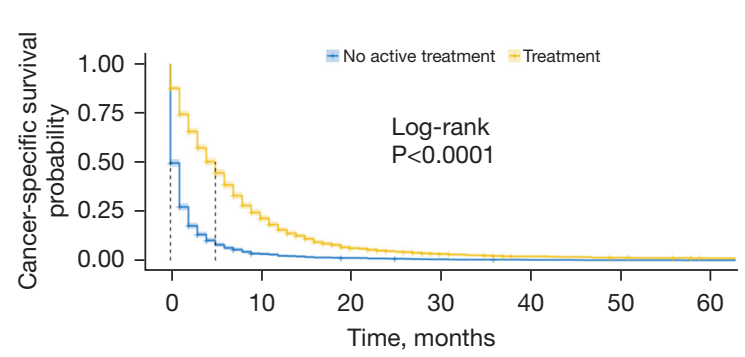
Number at risk

Number at risk

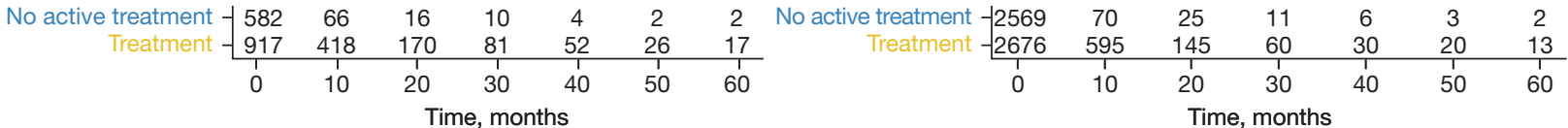

Figure 3 Cancer-specific survival by receiving active treatment in each stage. Survival curves were compared by a Log-rank test. (A) All patients; (B) patients in localized stage; (C) patients in regional stage; (D) patients in distant stage. The shaded part on the curve represents the $95 \% \mathrm{CI}$.

$0.33-0.42)$, which was even superior to chemotherapy only ( $\mathrm{HR}=0.58,95 \%$ CI: $0.46-0.74)$ without any overlap in CI.

\section{Discussion}

Medical treatment of elderly patients often presents clinicians' dilemmas for several reasons. Elderly patients usually have multiple comorbidities, poor performance status (PS), and short life expectancy (15), and increasing morbidity that limits potential benefits of various medications or interventions. Moreover, standardized treatment is usually based on randomized trials, which rarely include elderly participants, leaving the real life outcomes for this particular cohort largely unexplored. A systematic review analyzed 4,993 articles entailing metaor pooled-analyses of phase III, randomized controlled trials (RCTs), and found that participants $\geq 70$ years were underrepresented (16). However, patients with lung cancer tend to be diagnosed at an old age, and most
SCLCs are diagnosed with advanced stages and require multidisciplinary treatment (17) that may cause intolerable toxicity to the elderly. In those patients, the benefit and risk of each treatment option must be carefully evaluated to achieve an optimal balance. Previous studies revealed that octogenarians with NSCLC had a distinctive treatment profile (5-9). However, although they were less likely to be treated than the younger patients (7), those who did have received benefited from longer survival rates (6). Even with fewer data, the same results have been described for SCLC $(12,13)$. Therefore, this study aimed to verify the benefit of treatment and further investigate the role of different treatment methods in a stage-specific manner for octogenarians with SCLC.

Our results show that the treatment profile for elderly patients did not change much from 1988 to 2015 (Figure 1). However, despite the substantial proportion of octogenarians with SCLC not receiving active treatment (Figure 2), those who did have significantly improved survival (Figure 3), which 
A
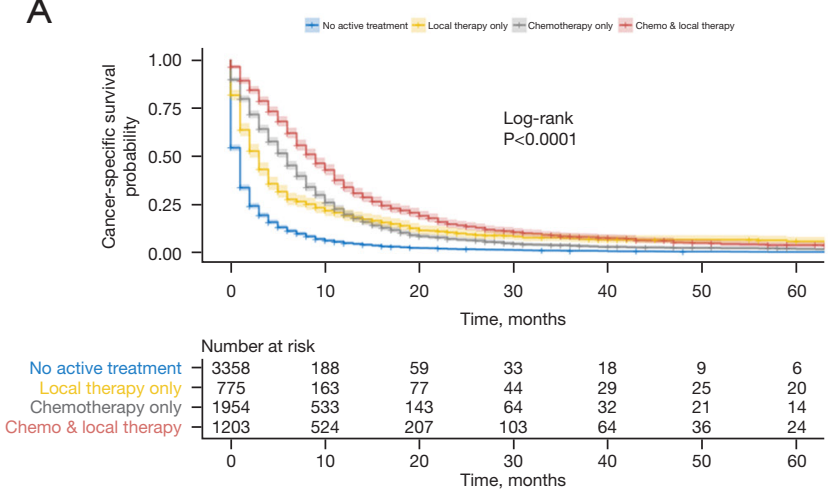

C
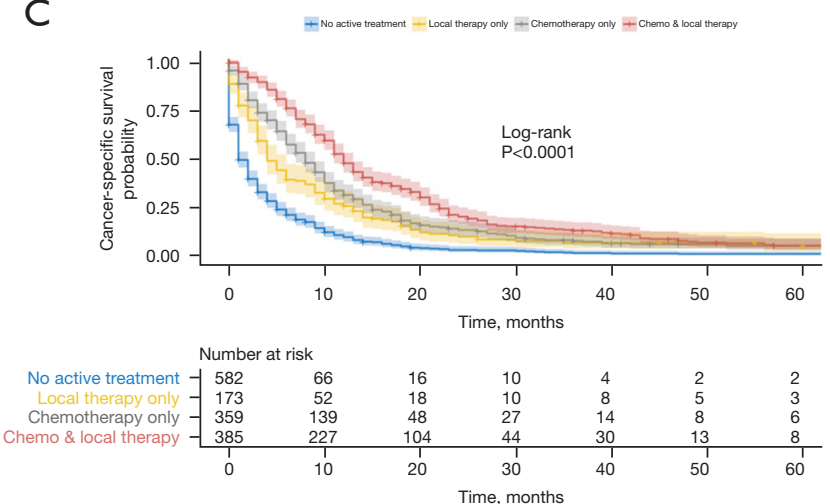

B
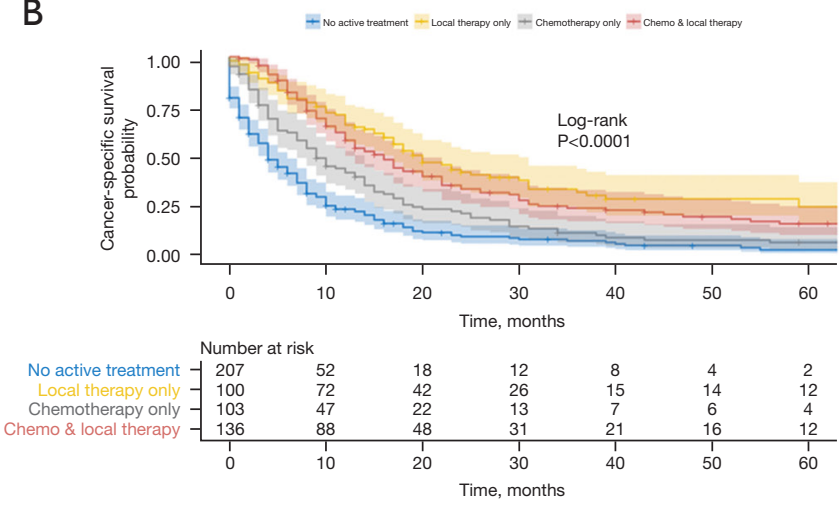

D
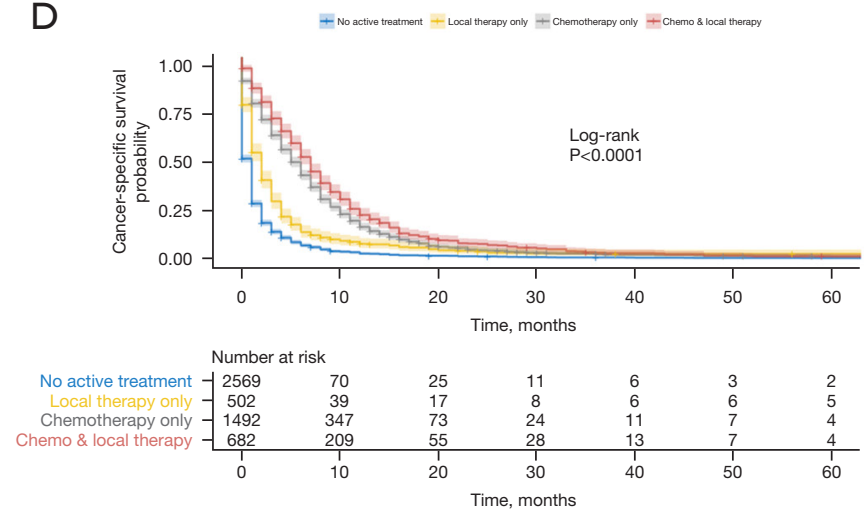

Figure 4 Cancer-specific survival by treatment methods in each stage. Survival curves were compared by a Log-rank test. All P values were less than 0.05 , except for local therapy only $v s$. chemo, and local therapy in localized stage for a pairwise comparison of survival difference between multiple groups. "BH" method was used to adjust the $\mathrm{P}$ value. (A) All patients; (B) patients in localized stage; (C) patients in regional stage; (D) patients in distant stage. Chemo \& local therapy is an abbreviation of chemotherapy and local therapy. The shaded part on the curve represents the $95 \% \mathrm{CI}$.

is true even after adjusting age, sex, race, and stage. Although treatment may be turned down for various reasons in the elderly patients, including the predicted poor prognosis of SCLC, increased risk of iatrogenic morbidities, economic or religious reasons, our results suggest we might be too conservative in the delivery of active treatment in the elderly patients with SCLC. This was most pronounced in the localized stage, whereas high as $38 \%$ of octogenarians were untreated, but treatment still resulted in survival benefit, increasing the median survival time by 9 months. Health care administrators and insurance companies are advised to include senior patients in the coverage, while clinicians should be more confident in offering treatment to the same group of patients.

When we look at each treatment method individually, chemotherapy-based treatment remained the gold-standard therapy with the best reduction in the risk of death for
SCLC, as is recommended by the guidelines (18). A study investigating patients 66 years or older with locally advanced NSCLC from SEER concluded that standard treatment based on randomized clinical trials could be extended to elderly patients (9). Our study suggested that the same rule may also apply to advanced SCLC. However, it is noteworthy that the benefit of chemotherapy may follow a stage-specific pattern, which has not been previously reported. When we evaluated the patients into subgroups with different stages, it was found that the benefit of chemotherapy declined as the stage became earlier. In the localized stage, the effect of chemotherapy only was significantly inferior to local therapy, and the combined therapy had a worse effect, although not statistically significant than local therapy only. The reversed ranking in the treatment effect suggests that local therapy may play a more critical role in treating octogenarians with SCLC in 
Table 2 Univariate and multivariate analysis for cancer-specific survival

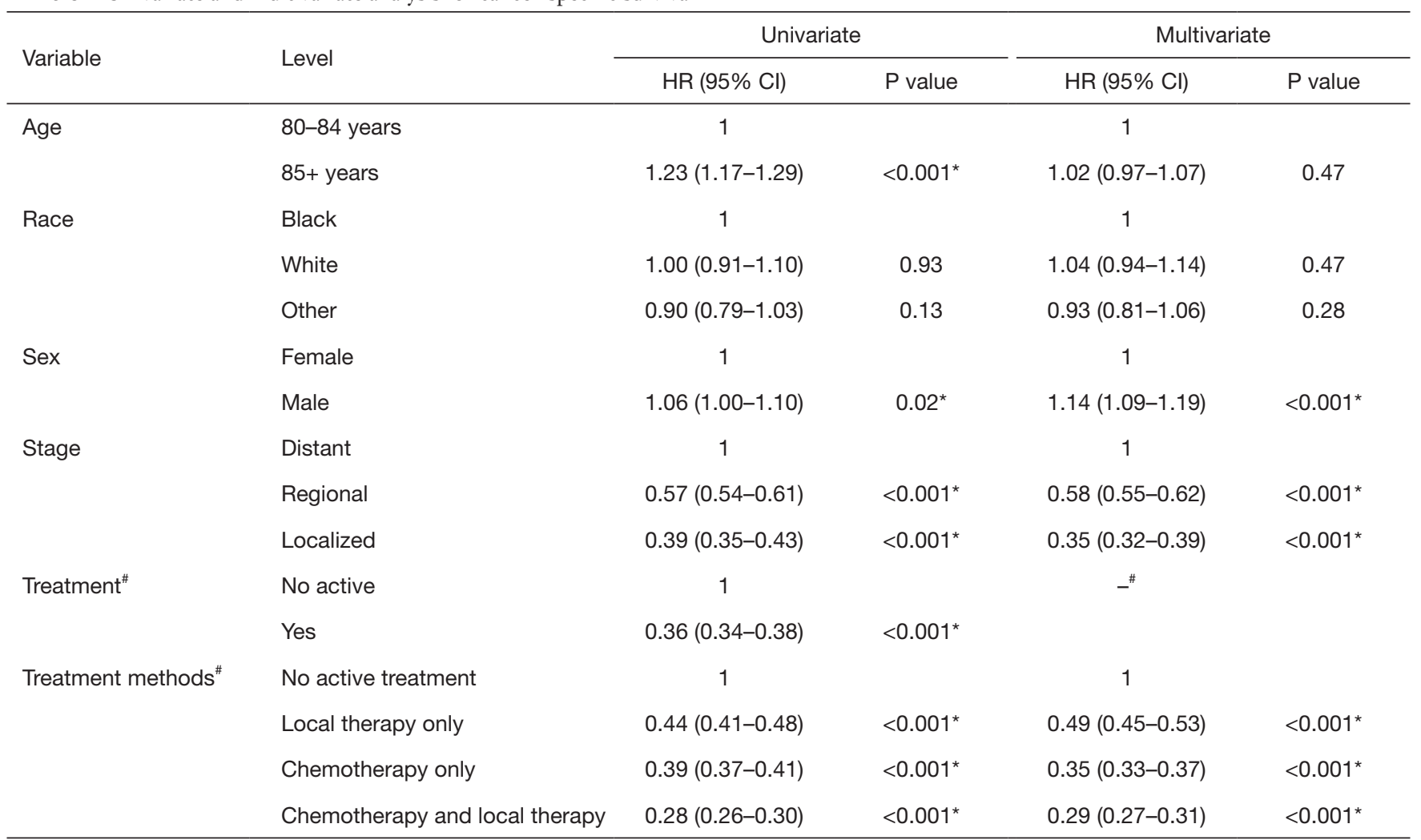

*, significant P values. \#, only treatment methods, but not treatment, were included in the multivariate analysis to avoid duplication. HR, hazard ratio; $\mathrm{Cl}$, confident interval.

Table 3 Multivariate analysis of cancer specific survival for localized, regional and distant stages

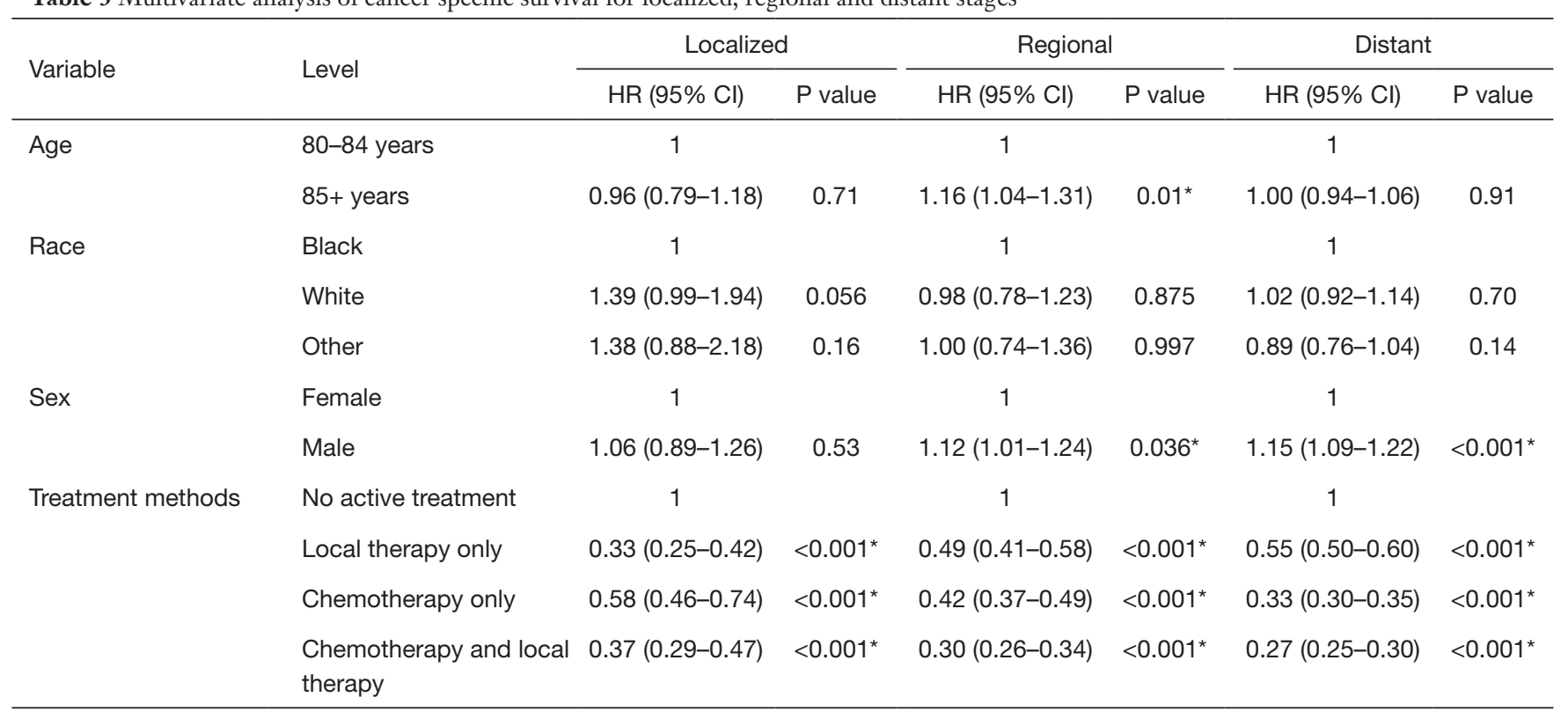

*, significant $\mathrm{P}$ values. HR, hazard ratio; $\mathrm{Cl}$, confident interval. 
the localized stage than in more advanced stages.

Local therapy was defined as surgery or radiotherapy, or both in our study. Although SCLC was historically described as a systemic disease, the role of local therapy has been increasingly realized in early-stage SCLC in most recent years (19-22). Surgery and stereotactic body radiation therapy is recommended for early-stage SCLC after a complete evaluation, according to ACCP guidelines $(18,19)$. In a review of the National Cancer Database (NCDB), for patients with node-negative SCLC, surgery with adjuvant therapy was associated with a higher OS than concurrent chemoradiation (5-year OS $49.2 \%$ vs. $32.5 \%, \mathrm{P}<0.01$ ) after propensity-score matching (20). For elderly patients aged 75-84 years with stage I SCLC, a study showed that surgery could significantly improve OS compared to non-surgical treatment (21). Albeit with rare incidence, asymptomatic SCLC manifesting as solitary pulmonary nodule without lymph node involvement, which would be easily mistaken for early-stage NSCLC, might represent a window early in its course that would benefit from surgical resection $(22,23)$. If such disease can be managed with minimal trauma, local therapy would be optimal for patients at a senior age by avoiding additional toxicity attributed to chemotherapy. Although surgery with adjuvant chemotherapy was favored over surgery alone for early-stage SCLC in a previous report (24), whether this is true for elderly patients may require further assessment. Radiotherapy usually refers to thoracic radiation therapy (TRT), and concurrent chemoradiotherapy (CRT) was widely applied as the standard treatment for LS-SCLC (25). Several studies also demonstrated the survival benefits of TRT after chemotherapy in patients with ES-SCLC (26-28). The efficacy and preference of TRT alone or surgery in the treatment of early-stage SCLC were still under debate $(25,29)$. Our results suggested that TRT may play a positive role in the treatment of early-stage SCLC.

Accumulating evidence shed light on the efficacy of immunotherapy and target therapy for SCLC in recent years. Pembrolizumab demonstrated the safety profile in the Phase Ib KEYNOTE-028 Study, which were applied to patients with ES-SCLC (30). In addition, maintenance pembrolizumab was used after induction chemotherapy for patients with ES-SCLC. Although patients did not improve median progression-free survival (PFS) compared with historical data, PFS and OS showed possible benefits (31). In another two phase 3 trials, both atezolizumab and durvalumab combined with chemotherapy improved OS in patients with ES-SCLC compared with chemotherapy alone $(32,33)$. In addition, a phase II study of anlotinib demonstrated an increase in progression-free survival (34). With the acceptable toxicity demonstrated by these reports, immunotherapy and target therapy may be promising options for elderly patients with SCLC.

There were some limitations in our study. First, it was a retrospective design with inherent selection bias. The baseline health or economic status was unavailable in the SEER but may affect treatment choice (35-37). Nevertheless, finding a better outcome for local therapy than chemotherapy in the localized stage will not be altered, as the latter would only be exempted for patients with compromised tolerance, leading to an only potentially healthier cohort than that of local therapy at baseline. Besides, since the conclusions were based on SEER database analysis, whether the conclusion was the same to Chinese octogenarians remained uncertain. Secondly, local therapy was not further divided into surgery or radiation, as the case number for each category was too small to justify a meaningful analysis. Further, studies with a larger sample size are warranted to illustrate the relative roles of surgery versus radiotherapy in detail. Thirdly, we used the historical stage of SEER (localized, regional, distant) instead of the recommended LS/ES stage or the TNM stage for SCLC. However, the general trend for the profile and benefit of different treatments kept the same as the disease progressed, and it has also been used this way in previously published articles $(38,39)$.

\section{Conclusions}

In conclusion, octogenarians may benefit from treatment for SCLC, yet nearly half did not receive active treatment in the real clinical setting. Advanced age ( $\geq 80$ years old) alone should not be used to exclude patient with SCLC from treatment. Standard treatment might be extended to octogenarians with advanced SCLC, while local therapy by itself may play a more important role than systemic therapies in treating octogenarians with localized SCLC.

\section{Acknowledgments}

The authors appreciate the academic support from AME Lung Cancer Collaborative Group.

Funding: This work was supported by the Cultivation Project of Shanghai Pulmonary Hospital (No. Fkcx1905, 
No. fk1911).

\section{Footnote}

Reporting Checklist: The authors have completed the STROBE reporting checklist. Available at https://dx.doi. org/10.21037/tlcr-21-839

Conflicts of Interest: All authors have completed the ICMJE uniform disclosure form (available at https://dx.doi. org/10.21037/tlcr-21-839). CSHN reports that he received consulting fees from Medtronic, Siemens Healthineer and Johnson \& Johnson for consulting services. The other authors have no conflicts of interest to declare.

Ethical Statement: The authors are accountable for all aspects of the work in ensuring that questions related to the accuracy or integrity of any part of the work are appropriately investigated and resolved. The study was conducted in accordance with the Declaration of Helsinki (as revised in 2013). The Institution Review Board of Shanghai Pulmonary Hospital approved this study.

Open Access Statement: This is an Open Access article distributed in accordance with the Creative Commons Attribution-NonCommercial-NoDerivs 4.0 International License (CC BY-NC-ND 4.0), which permits the noncommercial replication and distribution of the article with the strict proviso that no changes or edits are made and the original work is properly cited (including links to both the formal publication through the relevant DOI and the license). See: https://creativecommons.org/licenses/by-nc-nd/4.0/.

\section{References}

1. Bray F, Ferlay J, Soerjomataram I, et al. Global cancer statistics 2018: GLOBOCAN estimates of incidence and mortality worldwide for 36 cancers in 185 countries. CA Cancer J Clin 2018;68:394-424.

2. Gazdar AF, Bunn PA, Minna JD. Small-cell lung cancer: what we know, what we need to know and the path forward. Nat Rev Cancer 2017;17:725-37.

3. Lally BE, Urbanic JJ, Blackstock AW, et al. Small cell lung cancer: have we made any progress over the last 25 years? Oncologist 2007;12:1096-104.

4. Bade BC, Dela Cruz CS. Lung Cancer 2020: Epidemiology, Etiology, and Prevention. Clin Chest Med 2020;41:1-24.
5. Subramanian J, Morgensztern D, Goodgame B, et al. Distinctive characteristics of non-small cell lung cancer (NSCLC) in the young: a surveillance, epidemiology, and end results (SEER) analysis. J Thorac Oncol 2010;5:23-8.

6. Dalwadi SM, Szeja SS, Bernicker EH, et al. Practice Patterns and Outcomes in Elderly Stage I Non-Small-cell Lung Cancer: A 2004 to 2012 SEER Analysis. Clin Lung Cancer 2018;19:e269-76.

7. Gu Y, Zhang J, Zhou Z, et al. Metastasis Patterns and Prognosis of Octogenarians with NSCLC: A Populationbased Study. Aging Dis 2020;11:82-92.

8. Cassidy RJ, Zhang X, Switchenko JM, et al. Health care disparities among octogenarians and nonagenarians with stage III lung cancer. Cancer 2018;124:775-84.

9. Davidoff AJ, Gardner JF, Seal B, et al. Population-based estimates of survival benefit associated with combined modality therapy in elderly patients with locally advanced non-small cell lung cancer. J Thorac Oncol 2011;6:934-41.

10. Bei Y, Chen X, Raturi VP, et al. Treatment patterns and outcomes change in early-stage non-small cell lung cancer in octogenarians and older: a SEER database analysis. Aging Clin Exp Res 2021;33:147-56.

11. Abdel-Rahman O. Changing epidemiology of elderly small cell lung cancer patients over the last 40 years; a SEER database analysis. Clin Respir J 2018;12:1093-9.

12. Caprario LC, Kent DM, Strauss GM. Effects of chemotherapy on survival of elderly patients with smallcell lung cancer: analysis of the SEER-medicare database. J Thorac Oncol 2013;8:1272-81.

13. Schild SE, Zhao L, Wampfler JA, et al. Small-cell Lung Cancer in Very Elderly ( $\geq 80$ Years) Patients. Clin Lung Cancer 2019;20:313-21.

14. Benjamini Y, Hochberg Y. Controlling the False Discovery Rate: A Practical and Powerful Approach to Multiple Testing. J R Stat Soc Ser B Stat Methodol 1995;57:289-300.

15. Gosney MA. Clinical assessment of elderly people with cancer. Lancet Oncol 2005;6:790-7.

16. Dunn C, Wilson A, Sitas F. Older cancer patients in cancer clinical trials are underrepresented. Systematic literature review of almost 5000 meta- and pooled analyses of phase III randomized trials of survival from breast, prostate and lung cancer. Cancer Epidemiol 2017;51:113-7.

17. van Meerbeeck JP, Fennell DA, De Ruysscher DK. Smallcell lung cancer. Lancet 2011;378:1741-55.

18. Rudin CM, Ismaila N, Hann CL, et al. Treatment of Small-Cell Lung Cancer: American Society of Clinical Oncology Endorsement of the American College of Chest 
Physicians Guideline. J Clin Oncol 2015;33:4106-11.

19. Simone CB 2nd, Bogart JA, Cabrera AR, et al. Radiation Therapy for Small Cell Lung Cancer: An ASTRO Clinical Practice Guideline. Pract Radiat Oncol 2020;10:158-73.

20. Yang CJ, Chan DY, Shah SA, et al. Long-term Survival After Surgery Compared With Concurrent Chemoradiation for Node-negative Small Cell Lung Cancer. Ann Surg 2018;268:1105-12.

21. Li Y, Hu S, Xie J, et al. Effects of surgery on survival of elderly patients with stage I small-cell lung cancer: analysis of the SEER database. J Cancer Res Clin Oncol 2019;145:2397-404.

22. Yang CJ, Chan DY, Speicher PJ, et al. Surgery Versus Optimal Medical Management for N1 Small Cell Lung Cancer. Ann Thorac Surg 2017;103:1767-72.

23. Zhao X, Kallakury B, Chahine JJ, et al. Surgical Resection of SCLC: Prognostic Factors and the Tumor Microenvironment. J Thorac Oncol 2019;14:914-23.

24. Yang CF, Chan DY, Speicher PJ, et al. Role of Adjuvant Therapy in a Population-Based Cohort of Patients With Early-Stage Small-Cell Lung Cancer. J Clin Oncol 2016;34:1057-64.

25. Zhao S, Zhou T, Ma S, et al. Effects of thoracic radiotherapy timing and duration on progression-free survival in limited-stage small cell lung cancer. Cancer Med 2018;7:4208-16.

26. Tian S, Zhang X, Jiang R, et al. Survival Outcomes With Thoracic Radiotherapy in Extensive-Stage Small-Cell Lung Cancer: A Propensity Score-Matched Analysis of the National Cancer Database. Clin Lung Cancer 2019;20:484-493.e6.

27. Rathod S, Jeremic B, Dubey A, et al. Role of thoracic consolidation radiation in extensive stage small cell lung cancer: A systematic review and metaanalysis of randomised controlled trials. Eur J Cancer 2019;110:110-9.

28. Palma DA, Warner A, Louie AV, et al. Thoracic Radiotherapy for Extensive Stage Small-Cell Lung Cancer: A Meta-Analysis. Clin Lung Cancer 2016;17:239-44.

29. Misumi Y, Okamoto H, Sasaki J, et al. Phase I/II study of induction chemotherapy using carboplatin plus irinotecan and sequential thoracic radiotherapy (TRT) for elderly patients with limited-disease small-cell lung cancer (LDSCLC): TORG 0604. BMC Cancer 2017;17:377.

30. Ott PA, Elez E, Hiret S, et al. Pembrolizumab in Patients With Extensive-Stage Small-Cell Lung Cancer: Results From the Phase Ib KEYNOTE-028 Study. J Clin Oncol 2017;35:3823-9.

31. Gadgeel SM, Pennell NA, Fidler MJ, et al. Phase II Study of Maintenance Pembrolizumab in Patients with Extensive-Stage Small Cell Lung Cancer (SCLC). J Thorac Oncol 2018;13:1393-9.

32. Horn L, Mansfield AS, Szczęsna A, et al. First-Line Atezolizumab plus Chemotherapy in Extensive-Stage Small-Cell Lung Cancer. N Engl J Med 2018;379:2220-9.

33. Paz-Ares L, Dvorkin M, Chen Y, et al. Durvalumab plus platinum-etoposide versus platinum-etoposide in firstline treatment of extensive-stage small-cell lung cancer (CASPIAN): a randomised, controlled, open-label, phase 3 trial. Lancet 2019;394:1929-39.

34. Zhang C, Leighl NB, Wu YL, et al. Emerging therapies for non-small cell lung cancer. J Hematol Oncol 2019;12:45.

35. Pezzi TA, Schwartz DL, Mohamed ASR, et al. Barriers to Combined-Modality Therapy for Limited-Stage Small Cell Lung Cancer. JAMA Oncol 2018;4:e174504.

36. Rich AL, Tata LJ, Free CM, et al. How do patient and hospital features influence outcomes in small-cell lung cancer in England? Br J Cancer 2011;105:746-52.

37. Zikos E, Ghislain I, Coens C, et al. Health-related quality of life in small-cell lung cancer: a systematic review on reporting of methods and clinical issues in randomised controlled trials. Lancet Oncol 2014;15:e78-89.

38. Komiya T, Guddati AK, Nakanishi Y. Clear cell adenocarcinoma of the lung: a SEER analysis. Transl Lung Cancer Res 2019;8:187-91.

39. Zhou H, Xian W, Zhang Y, et al. Trends in incidence and associated risk factors of suicide mortality in patients with non-small cell lung cancer. Cancer Med 2018;7:4146-55.
Cite this article as: Zhou B, Li Q, Qin L, Li Z, Jin K, Dai J, Zhu Y, Yang Y, Jabbour SK, Tartarone A, Ng CSH, Navarro A, Pompili C, Jiang G. Octogenarians may benefit from stagespecific small cell lung cancer treatment. Transl Lung Cancer Res 2021;10(10):3973-3982. doi: 10.21037/tlcr-21-839 